\title{
A Hybrid CNN-LSTM Based Approach for Anomaly Detection Systems in SDNs
}

\author{
Mahmoud Said Elsayed \\ University College Dublin, Ireland \\ mahmoud.abdallah@ucdconnect.ie \\ Hamed Z. Jahromi \\ University College Dublin, Ireland \\ hamed.jahromi@ucdconnect.ie
}

\author{
Nhien-An Le-Khac \\ University College Dublin, Ireland \\ an.lekhac@ucd.ie \\ Anca Delia Jurcut \\ University College Dublin, Ireland \\ anca.jurcut@ucd.ie
}

\begin{abstract}
Software-Defined Networking (SDN) is a promising technology for the future Internet. However, the SDN paradigm introduces new attack vectors that do not exist in the conventional distributed networks. This paper develops a hybrid Intrusion Detection System (IDS) by combining the Convolutional Neural Network (CNN) and Long Short-Term Memory Network (LSTM). The proposed model is capable of capturing the spatial and temporal features of the network traffic. Two regularization techniques i.e., L2 Regularization (L2 Reg.) and dropout method are used to overcome with the overfitting problem. The proposed method improves the intrusion detection performance of zero-day attacks. The InSDN dataset - the most recent dataset for SDN networks is used to test and evaluate the performance of the proposed model. The results indicate that integrating the CNN with LSTM improves the intrusion detection performance and achieves an accuracy of $96.32 \%$. The estimated accuracy is higher than the accuracy of each individual model. In addition, it is established that the regularization techniques improves the performance of the $\mathrm{CNN}$ algorithms in detecting new intrusions when compared to the standard CNN. The findings of this study facilitates the development of robust IDS systems for SDN environment.
\end{abstract}

\section{KEYWORDS}

CNN, Deep Learning, InSDN, Intrusion Detection System, LSTM

\section{ACM Reference Format:}

Mahmoud Said Elsayed, Nhien-An Le-Khac, Hamed Z. Jahromi, and Anca Delia Jurcut. 2021. A Hybrid CNN-LSTM Based Approach for Anomaly Detection Systems in SDNs. In The 16th International Conference on Availability, Reliability and Security (ARES 2021), August 17-20, 2021, Vienna, Austria. ACM, New York, NY, USA, 7 pages. https://doi.org/10.1145/3465481.3469190

\section{INTRODUCTION}

Software-Defined Networking (SDN) is a networking model that provides network flexibility and programmability through a central control plane. The principal concept of SDN is to separate the

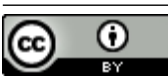

This work is licensed under a Creative Commons Attribution International 4.0 License.

ARES 2021, August 17-20, 2021, Vienna, Austria

(C) 2021 Copyright held by the owner/author(s).

ACM ISBN 978-1-4503-9051-4/21/08

https://doi.org/10.1145/3465481.3469190 control plane (decides how data packets are forwarded) from the data plane (forwards the packets). In a SDN environment, the entire network is managed from a centralized control point, i.e. SDN controller. The SDN controller has a global overview of the network and performs a network-wide policy enforcement. SDN has been a driver of several applications, including Quality of Service (QoS), load balancing, network monitoring, threat detection and prevention $[9,17,27]$. The global view of the network environment allows to understand the current state of the network. The network heuristics and traffic flows can be monitored and observed from a centralized point. However, in conventional networks and due to the distributed nature of the environments, accessing network heuristics and traffic flow information are a challenging task and can utilize a high percentage of computation resources since it involves all the network nodes. Therefore, the SDN technology received significant attentions from several global enterprises and service providers including Google, Facebook, and Microsoft. SDN is now widely adapted and deployed in production environment (e.g., dacanters) [23]. However, the security in SDN is a doubleedged sword. The centralized architecture of SDN provides better network visibility, supports security and threat detection applications. Nevertheless, separating the control plane from the data plane creates new attack vectors that do not exist in the distributed networks. In addition, the popular attack vectors in conventional networks are also common in SDN, but with severe consequences as compared to other networks $[5,16]$. For example, Distributed Denial of Service (DDoS) attacks can only impact a portion of the distributed networks. However, the same attacks towards a SDN controller can have serious impact and can interrupt the SDN controller (single point of failure) and the network functionality in very short time $[2,9]$. Hence, protecting the SDN environment from malicious attacks is crucial to fully unleash the capabilities of the new model.

Intrusion Detection Systems (IDSs) are essential tools to protect and secure the network from malicious attacks. Two popular techniques have been adopted for IDS: signature-based detection and anomaly-based detection approaches. While signature-based detection experiences high detection rates and low false alarms, it fails to detect the zero-day attacks. The second approach, anomaly-based detection, theoretically can detect novel attacks that have never been seen before; however, such techniques are still experiencing high false alarms. Hence, further improvement of the anomalybased detection approaches is essential to enhance SDN network security. 
The contribution of this paper includes the following:

- We propose a hybrid IDS by combining the Convolutional Neural Networks (CNN) with Long Short Term Memory (LSTM) to learn the spacial and temporal features from the input traffic. We use $L 2$ Reg. and dropout regularization techniques during the training of the IDS model in order to reduce the impact of overfitting problem. Hence, our model has a significant performance in detecting the new intrusions, which are not being seen during the training.

- We train and evaluate our proposed IDS model in a SDN environment, i.e. we use the most recent available SDN attack specific dataset, InSDN [8].

- We use various evaluation metrics i.e., accuracy, precision, recall, F1-score and area under curve, to evaluate the performance of our model. The results show that our proposed hybrid CNN-LSTM model has a higher performance in terms of all evaluation metrics when compared to individual models.

The rest of this paper is organised as follows: Section 2 provides a brief background about the CNN, LSTM, the overfitting problem and regularization methods. Section 3 gives an overview of the existing machine learning (ML) and deep learning (DL) techniques that are currently used to monitor and detect threats in SDNs. The proposed model including the evaluation dataset and the experimental setup are provided in Section 4. The obtained experimental results are discussed in section 5. Finally, Section 6 and 7 discusses and concludes the paper, respectively.

\section{BACKGROUND}

\subsection{Intrusions and Anomalies}

The words of intrusions and anomalies are commonly used interchangeably in the context of IDS; however, both terms are quite confusing. The intrusion is the malicious activities that attempt to compromise the confidentially, availability, and integrity, while anomaly refers to patterns in data that do not conform to the expected normal behaviour, i.e. deviation from what is considered as normal. However, the notion of anomaly depends on the application domain and context, i.e. anomaly is not always an intrusion. For example, in the network domain, network traffic, performance indices and logs are monitored to detect failures in the network (it is not an intrusion). The applications of anomaly detection have extended to diverse areas such as suspicious movements in video surveillance [10], sensors failures in industry [19], life-threatening situations in medical applications [25], and credit card fraud in fraud detection domain [14]. Nevertheless, IT administrators in the cybersecurity domain narrow down the definition of anomaly and they consider any events that deviate from normal behavior as potential intrusion attempts. In the other way, the system that tries to detect intrusions is also a general term for the system that tries to find anomalies. Anomaly detection approaches can be divide based on the training method used into statistical and machine-learning [26].

\subsection{Convolutional Neural Networks (CNN)}

CNN belongs to the supervised learning strategy and is one of the robustness algorithms widely applied in computer vision. $\mathrm{CNN}$ can speed the training process since it solved the problem of parameter explosion by introducing the weight sharing concept. It has been used in various applications, such as face reorganization, image pre-processing. The architecture of the $\mathrm{CNN}$ composites from three main components, namely convolution layer, pooling layer, and fully connected layer. A simple architecture of the CNN is depicted in Fig. 1. The convolution layer applies the linear operation by moving the filter (kernel) with a specific size through the output of the previous layer. The non-linear Relue function is the dominant activation function widely used with $\mathrm{CNN}$ to increase the non-linearity degree and to set all negative values in the feature map by zero. The $\mathrm{CNN}$ can contain more than a single convolution layer, where the first convolution layer is almost used to extract the simple features like edges and corners, while the next layers are used to extract the complex features. The pooling layer is used to reduce the feature dimensions, and hence it can minimize the computational cost. The last fully connected layer is used for classification purposes.

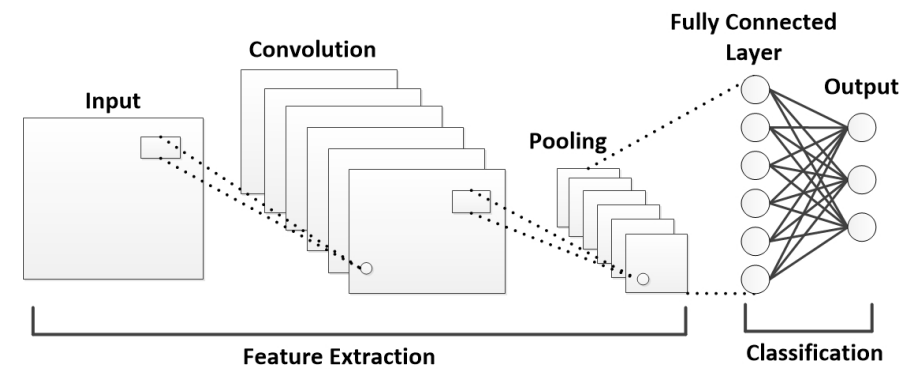

Figure 1: A typical architecture of the CNN

\subsection{Long short term memory (LSTM)}

LSTM is a special form of recurrent neural network (RNN) and broadly applied for processing time-series data. In the standard $\mathrm{RNN}$, the output of any layer is not only dependent on the current input but also based on the previous output. However, one of the main challenges to train the standard RNN is the problem of the vanishing gradients. Gradients are used to update the weight values of a neural network, as shown in equation 1 . However, when a gradient value becomes extremely small, as it back propagates through time, it does not contribute too much learning. The RNN suffers from small gradient updates, especially in the earlier layers. Thus, it is unable to keep the information for the long sequences.

$$
\text { New weight }=\text { weight }- \text { learning rate } * \text { gradient }
$$

LSTM [13] was introduced to solve the vanishing gradient, and therefore it is more suitable for long-time step preprocessing. LSTM produces the concept of internal loops to keep only the useful information and discards non relevant data. LSTM has three different gates i.e., forget gate, input gate, and output gate to regulate information flow in each LSTM cell. The interested reader may refer to [18] for more details regarding the computation process of the LSTM.

We use the LSTM in this work because the temporal correlation of the network traffic generates time-series data [24]. In addition, 
the CNN has achieved good results in image processing due to its ability to learn spatial features. Thus, integrating the CNN with LSTM can significantly extract the spatial and temporal attributes of the raw data and therefore improve the accuracy of the intrusion detection system.

\subsection{Overfitting and Regularization}

Overfitting is a major problem in neural networks and most of time occurs when the model performs very well on training data, while it has a very bad intuition on the testing. This happens when the model learns the details and noise (e.g., outliers) of the training data but fails to generalize the scenario when it is used for evaluating new data points. There are several ways to reduce the problem of overfitting. A common approach to avoid overfitting is to increase the amount of training data since the model is unable to overfit all samples and it will be forced to generalize in order to obtain good results. However, this method is considered expensive and constrained by the availability of datasets (it can be a significant challenge), especially for the network traffic. The network traffic can contain customer information and the availability of such datasets can espouse personal information to the public. An alternative solution is to use regularization techniques to add the penalty for the higher weights. It can thus reduce the model complexity. Several regularization methods, i.e. $L 1$ and $L 2$ can be used to optimize the neural network model and to encourage smaller weights. Besides the aforementioned regularization methods, the dropout is also a commonly used technique to reduce the risk of overfitting. In the dropout, we randomly ignore some neurons from the network with a probability of $P$, but all discarding units are used during the testing phase.

\section{RELATED WORK}

Nowadays, the IDSs have gained significant attention of researchers from academia and industry. Machine learning (ML) techniques have been mainly used in IDSs and are proved to be effective for detecting anomalous activities [1, 4, 6, 11, 22]. For instance, algorithms such as Logistic Regression (LR), Decision Trees (DTs), Naïve Bayes (NB), and Support Vector Machine (SVM) are widely used to identify various network-based attacks. These aforementioned techniques are known as shallow learning due to its learning characteristics based on pre-defined features. Selecting the best features is not an easy task since the features that might be important for one attack class might not be essential or applicable for different classes. In addition, these techniques reported high false alarm rates and require detailed knowledge of the problem domain. Moreover, developing discriminative features methods is not beneficial when only normal data is available. Further, the ML techniques provide high performance when the labeled data has a small sample size. Such techniques suffer from several limitations and are not significant when applied on huge network traffic data.

Deep Learning (DL) is a subset of ML which has been successfully applied in many research applications such as speech recognition, natural language processing, and image processing. The advantage of DL is that it does not need the feature extraction step. It has a capability to learn the implicit representation of the raw data automatically without prior expert knowledge. In recent years, several DL approaches have been applied for IDSs. The DL has a more robust ability to learn the deep structure from raw data and obtain the features automatically, instead of using manual feature selection and extraction.

In [7], Elsayed et al. used DL to solve the problem of the minority attacks in imbalanced datasets. The proposed model integrated the autoencoder with LSTM in unsupervised learning. The model was trained using only samples from the normal data, and then it was applied for a new testing data, which included both normal and malicious traffic. Since the normal traffic is only used due to the training phase, the model is able to reconstruct the input at the autoencoder output with very low reconstruction error. In contrast, this error is relativity high in testing, as it has samples from both normal and malicious traffic. The reconstruction error is used as a threshold to separate between the different boundaries, i.e. normal and malicious. However, the model only provided significant results in old datasets (i.e. the NSL-KDD dataset), but fails to perform well with the most recent datasets. This is due to fact that the recent datasets contain sophisticated attacks which have a high degree of similarity with normal traffic. Thus, the simple threshold is unable to comprehensively distinguish the various network traffic classes.

To overcome with the limitation of a simple threshold in the aforementioned work, the authors integrated the One-class SVM (OC-SVM) with the LSTM-Autoencoder [21] in unsupervised learning. The LSTM-Autoencoder was used in the early stage to extract the compressed features (i.e. latent) from the input data. Then, the extracted features are used to train another OC-SVM to detect anomalies in the testing data. The experimental results show that combining the OC-SVM with DL approaches can effectively improve the performance of the detection model when compared to the single OC-SVM algorithm.

Tang et al. [23] applied the Deep Neural Network (DNN) for flow-based anomaly detection in the SDN network. Only six basic features were utilized from the NSL-KDD dataset to reduce the computational cost of intrusion detection. The structure of the DNN model is composited from three hidden layers with a number of neurons equal to 12,6 , and 3, respectively. The proposed model provided an overall accuracy of $75 \%$, which is below a threshold that can be deployed in a real environment. The authors enhanced their proposed model by employing Gated Recurrent Unit (GRU) to achieve better accuracy using the same NSL-KDD dataset [24]. The detection rate of their enhanced model is improved to $89 \%$.

Boukria et al. [3] proposed an anomaly-based approach to detect different attacks in SDN networks. The DNN approach constructed from three hidden layers with neuron dimensions equal to 128,64 , and 32, respectively. The authors claim that the model provided an overall accuracy of $99.6 \%$ using the CICIDS2017 dataset, as compared to other state-of-art solutions.

However, the previous DL techniques requires a large number of training parameters (since all adjacent layers are fully connected together). Using a high number of parameters can slow down the training process and increase the computational cost of the detection model. Thus, adds extra computational overhead in a SDN environment.

Motivated by the successful application of the CNN-based network modeling for several challenging classification problems, our 
proposed IDS applies the CNN model for efficient and early detection of SDN network attacks. Besides, dimensionality reduction is a factor that inspired us to use $\mathrm{CNN}$. Although the $\mathrm{CNN}$ has been used for anomaly detection [15, 28], it fails to give reasonable results in anomalies discovery. The malicious traffic exhibits a high degree of similarity with normal data, with only small variation between them. CNN should be adopted to distinguish the little differences between different boundaries. To address this problem, we use regularization techniques in the presented study to reduce the overfitting and to create a generalized model that is capable to fit well on unseen datasets or unknown instances. Thus, we can improve the performance of the CNN on anomaly detection. In addition, we combined the CNN with LSTM to extract the spatial and temporal features from the input data. To the best of our knowledge, there is no prior work conducted the CNN with LSTM for anomaly detection in SDN networks.

\section{PROPOSED MODEL}

\subsection{Dataset}

We use a very recent updated SDN attack-specific dataset, i.e. InSDN [8] to evaluate the performance of our proposed model. The InSDN dataset is considered one of the first solutions to produce a comprehensive dataset, available to evaluate the IDSs for SDNs. The dataset has unique attack characteristics that are specific to SDN network. Selecting a SDN specific dataset is essential for properly evaluating SDN-based attack detection approaches. Using non-specific SDN datasets can cause a compatible problem, since the attack vectors deployment should consider the new architecture of the network [20]. In addition, the working principles of some attacks differ on SDN networks compared to conventional networks. For example, the intruder can utilize some attacks like "IPsweep" and "Portscan" to overwhelm the SDN controller resources with unknown packets causing a DDoS attack. Although the aforementioned attacks are not a type of DDoS according with conventional detection techniques, they can be used to generate an extensive amount of network flows in the SDN network.

The InSDN dataset contains several types of normal traffic application (HTTP, HTTPS, DNS, Email, FTP and SSH), the common attacks that exist in conventional networks and the SDN specific attacks. The attack types include DoS, DDoS, Probe, Botnet, Exploitation, Password-Guessing, and Web attacks. In addition, these attacks come from several sources, internal and external, to reflect the real attack cases.

The dataset is available in both PCAP file and .CSV file format and is divided into three groups. The first group OVS group, includes the attacks coming from outside to SDN internal network. The second group contains the attacks against Metasploitable 2 server. The last group represents the normal traffic. The dataset has more than 80 features generated using the CICFlowMeter tool.

In this work, we only use a subset of 48 features to train our CNN model. More details about these features can be found in [8]. To test the sufficiency of our proposed model for anomaly detection, we used attack samples in the testing phase that are different from those used during the training. The data records for training and testing datasets are 133,334 and 74,343, respectively.

\subsection{Dataset Preparation}

The following pre-processing steps are performed before feeding the input data into the proposed model:

(1) The normalization technique is applied to map the value of features between 0 and 1 , using the standardization method (Z-score normalization) according to the equation 2.

$$
\mathrm{x}(\mathrm{i})=\frac{x(i)-\operatorname{mean}(x(i))}{\text { standard_deviation }(x(i))}
$$

Where, i $\epsilon[1,48]$

(2) We used 2D-CNN instead of 1D-CNN for our approach to reduce the number of weights within a convolution layer through the parameter sharing concept. However, network data is not an image, so a further step is initially required to convert the network data into an image structure. The 1D-dimensional 48 network traffic features are translated into image format with a dimension of $8 \times 6$ to fit with the $\mathrm{CNN}$ input requirements.

(3) The symbolic features are converted into numerical data. The label column has several attack classes as well the normal class. It is also important to note that this study preforms a binary classification. Therefore, we set the normal label to 0 and all attack labels have been assigned to 1 .

\subsection{Experimental Setup}

The Keras ${ }^{1}$ Library with Python programming language was used to perform all our experiments. The experiments are achieved on a machine with Intel core I7-8650U CPU @ $1.90 \mathrm{GHz}$ (8 cores), Window 10 operating system. The InSDN is the benchmark dataset used for all classification experiments to perform 2-class anomaly detection.

\subsection{Building our model}

This section introduces the architecture of our hybrid model to learn and classify the network traffic in both space and time. The overall network architecture of the model is depicted in Fig. 2. The architecture is composed of two stages.

In the first stage, the $\mathrm{CNN}$ is used to extract the spatial features and contains two convolution layers with output dimensions of 32 and 64, respectively. The kernel with size of $3 \times 3$ is used for both convolution layers. Each convolution layer is followed by a Maxpooling layer with a dimension of $2 \times 2$ to reduce the dimensionality of map features.

The high-dimensional features extracted from the CNN stage are fed into the second stage, which is composed of three layers including: the LSTM layer, the fully connected layer, and the output layer. The number of nodes in LSTM and fully connected layer is 128 for each. Finally, the softmax layer is used at the output for the classification purposes, to represent the probability of each input flow.

We used $L 2 R e g$. and dropout methods to reduce the effect of overfitting and to enhance the capability of the detection model in unseen data. We conducted several experiments to select the best regularization hyper-parameter $\lambda$ as long the probability values of

\footnotetext{
${ }^{1}$ The Keras Library is available on: https://keras.io/
} 


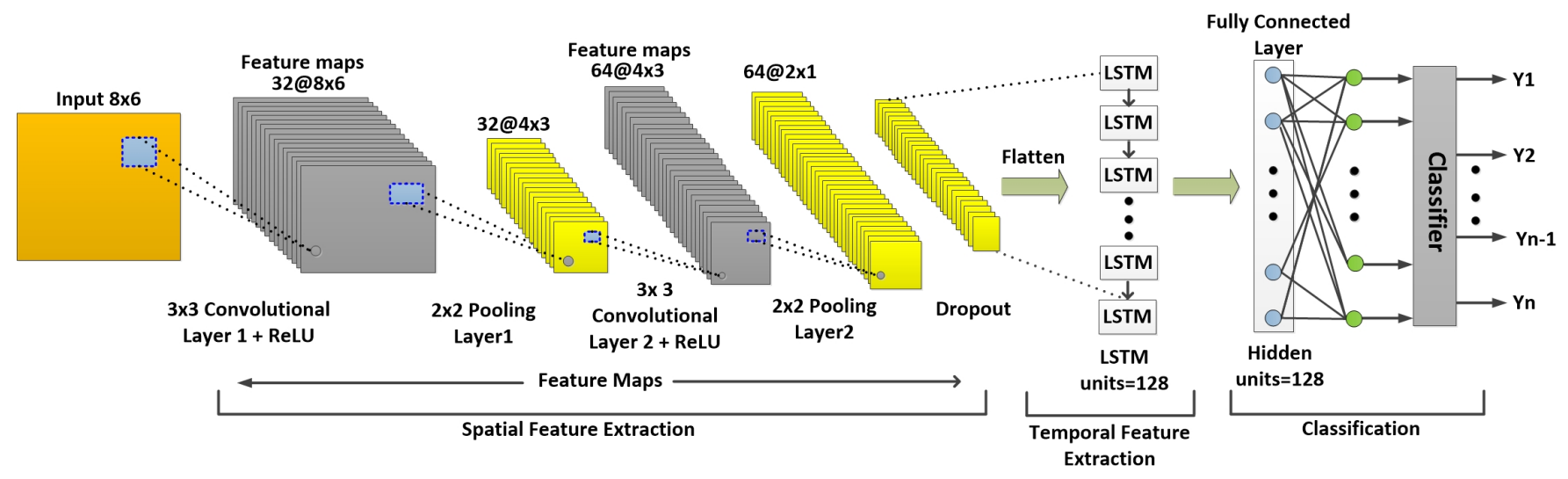

Figure 2: A typical architecture of the proposed CNN-LSTM model.

the dropout technique. The value of $\lambda$ which is used for $L 2$ Reg. is set to 0.1 , while the dropout with the probability $P$ of 0.25 and 0.5 are used after the second pooling layer and the fully connected layer, respectively. We start with small value of dropout probability and then gradually increase it, since the dropout can cause some information loss inside the learning model, and we aim to reduce the propagation of this loss to the next layers. In this experiment, we only provide a binary classification, where the softmax output belongs to the normal or attack class.

\section{EVALUATION RESULTS}

This section discusses the experimental results of the proposed model.

\subsection{Evaluation Criteria}

To evaluate our CNN model, four performance indicators of Accuracy (Acc), Precision (Pre), Recall (Rec) and F1 measure are used. The mathematical representation of these indicators are calculated based on equations $3,4,5$, and 6 , respectively.

$$
\begin{gathered}
\text { Acc }=\frac{T P+T N}{T P+T N+F P+F N} \\
\text { Pre }=\frac{T P}{T P+F P} \\
\text { Rec }=\frac{T P}{T P+F N} \\
\mathrm{~F} 1=\frac{2 \times \text { Pre } \times \text { Rec }}{\text { Pre }+ \text { Rec }}
\end{gathered}
$$

TP, TN, FP and FN denote true positives, true negatives, false positives, and false negatives, respectively.

\subsection{Experimental Results}

The evaluation results are represented in Table 1, Fig. 3, Fig 4. and Fig. 5. It can be seen that our proposed CNN-LSTM model performs well compared to the other existing methods. Table 1 and Fig 4 show that the standard $\mathrm{CNN}$ algorithm has a low performance compared to other DL models. The average accuracy of the standard $\mathrm{CNN}$ is $90.79 \%$; while when we use the regularization methods,
Table 1: Precision, Recall, and F1-score of the different methods.

\begin{tabular}{|l|l|l|l|l|l|l|}
\hline \multirow{2}{*}{ Model } & \multicolumn{2}{|c|}{ Precision (\%) } & \multicolumn{2}{c|}{ Recall (\%) } & \multicolumn{2}{c|}{ F1-Score (\%) } \\
\cline { 2 - 7 } & Normal & Attack & Normal & Attack & Normal & Attack \\
\hline CNN Standard & 76.69 & 98.86 & 97.47 & 88.11 & 85.84 & 93.18 \\
\hline LSTM & 84.53 & 98.31 & 96.02 & 92.95 & 89.91 & 95.55 \\
\hline CNN (L2 Reg.) & 84.24 & $\mathbf{9 8 . 5 6}$ & $\mathbf{9 6 . 6 2}$ & 92.75 & 90.00 & 95.56 \\
\hline CNN-LSTM & $\mathbf{9 3 . 1 8}$ & 97.60 & 94.04 & $\mathbf{9 7 . 2 4}$ & $\mathbf{9 3 . 6 1}$ & $\mathbf{9 7 . 4 2}$ \\
\hline
\end{tabular}

the performance of $\mathrm{CNN}$ is significantly increased, and scored an accuracy of $93.83 \%$. In addition, the performance of LSTM is relativity higher compared to the standard CNN but quite less than the $\mathrm{CNN}$ with regularization. Furthermore, combining the CNN with LSTM outperforms all other algorithms - the accuracy obtained is $96.32 \%$, which proves the effectiveness of the proposed hybrid CNN-LSTM model for intrusion detection. On the other side, we can see that the CNN (L2 Reg.) model has a higher precision for the attack class and a higher recall for normal class when compared to all other algorithms. However, the CNN-LSTM model provides the best F1-score for the two classes.

Additionally, we report the performance of our proposed method for the classification of normal and attack data. The confusionmatrix (CM) of different DL approaches obtained from the testing stage is illustrated in Fig. 3. Each event in the testing set either belong to normal or attack events. Our proposed CNN-LSTM model indicates a higher degree of accuracy (0.97) in correctly detecting the attack events. However, the accuracy is slightly lower at 0.94 for normal traffic events. This is due to the low normal samples which are used during the training and testing phases, compared to the attack classes.

To further evaluate our proposed CNN-LSTM model, the receiver operating characteristics (ROC) curve (Fig. 5) is used to represent how the detection model performs in general. The ROC curve represents the relation between the true-positive and false-positive rates, and the area under the curve (AUC) is used to measure the model's capability. The proposed CNN-LSTM model has a higher AUC with a value of 0.956 , followed by the CNN ( L2 Reg.) and the LSTM algorithms with the values of 0.947 and 0.945 , respectively. In 


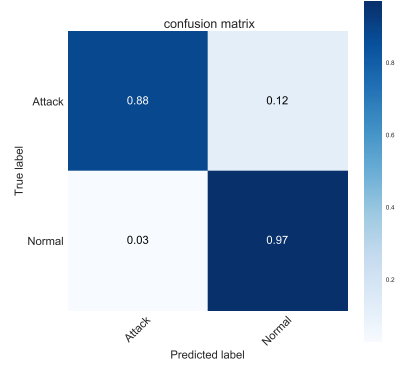

(a) CNN (Standard)

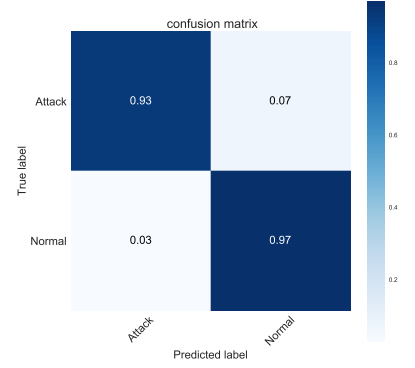

(b) CNN (L2-Reg.)

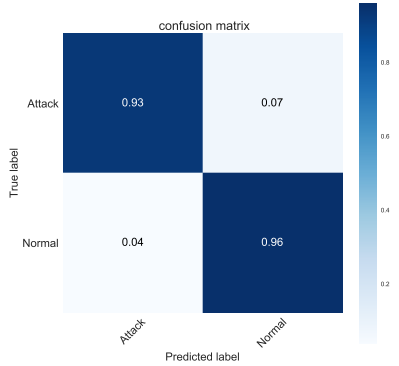

(c) LSTM

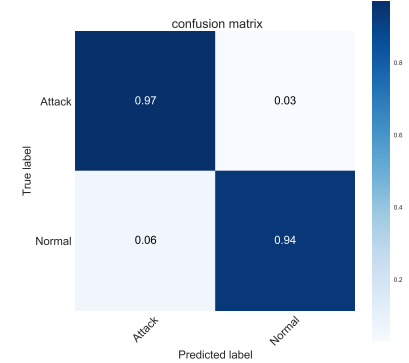

(d) CNN-LSTM

Figure 3: Confusion matrix for Binary classification obtained from DL approaches.

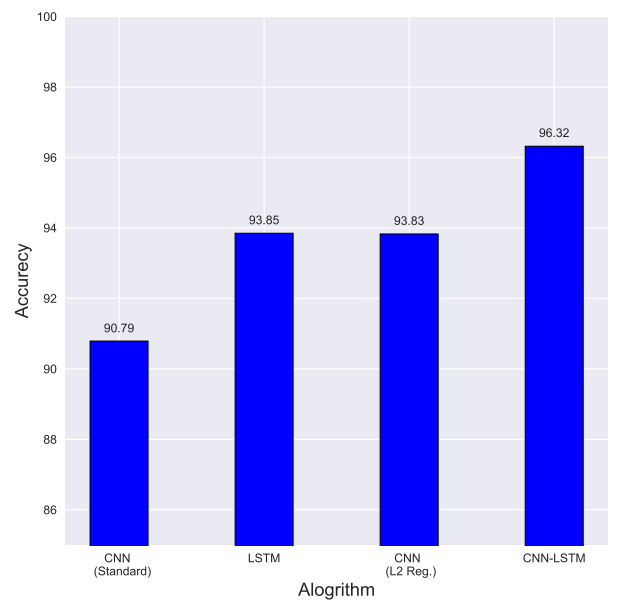

Figure 4: Comparison of the different approaches and other algorithms

contrast, the standard CNN provides the lowest AUC value (0.928), which indicates a lower performance of this algorithm for network anomaly detection.

\section{DISCUSSION AND LIMITATION}

In this article, we proposed a hybrid model by integrating the CNN with LSTM algorithms in order to improve the SDN capability to detect the malicious network activities. One of the main challenges in the training of ML/DL is the risk of overfitting, where the model performs very well during training, but it fails to generalize smoothly in the new instances. This problem almost occurs when the training data has a large amount of noise or outlier, which does not really represent the actual properties of the regular patterns. To overcome this problem, the L2 regularization and dropout techniques have been conducted with the hybrid model to enhance the performance

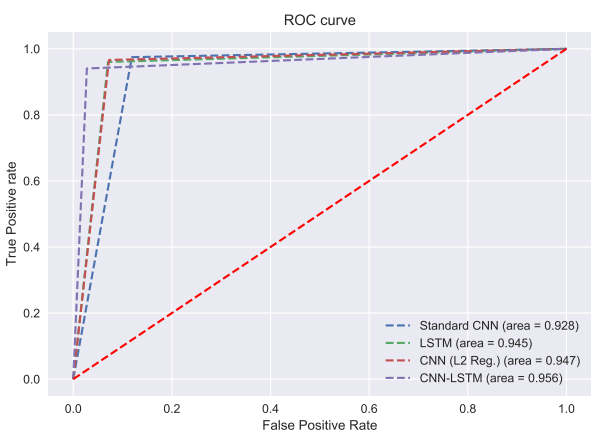

Figure 5: Receiver Operating Curve (ROC) of our proposed approach.

of the DL model in the new attacks that were never seen before during the training. We tested the functionality of the hybrid model by selecting the attack instances in the testing data with different distributions from those used during the training. The reported results indicated that the proposed DL model achieved a good performance and hence it can be applied for zero-day attack detection . In addition, the $2 \mathrm{D}-\mathrm{CNN}$ was used in our experiments to reduce the number of the training parameters compared to $1 \mathrm{D}-\mathrm{CNN}$. This is because the $2 \mathrm{D}-\mathrm{CNN}$ produces the concept of weight sharing, and hence the computational cost can dramatically become less.

Likewise, it can be seen that the false alarms of the proposed model are quite high. The percentage of false positive rate (FPR) and false negative rate (FNR) of the hybrid model are $6 \%$ and $3 \%$, respectively. The highest false alarms can prevent the real deployment of the learning model in the production environments. However, the training process of the model has been done in supervised learning. Although the supervised learning can successfully provide a detection rate of over $99 \%$, it fails to provide acceptable results for the new attack instances. It is similar to signature-based detection algorithms in its operation and requires the same baseline of training data during the testing. Hence, many research approaches 
rely on unsupervised learning techniques to enhance the performance of the detection model for zero-day attacks [12]. However, unsupervised learning is still immature for real implementation deployments, since it yields to high false alarms and low detection rate. Moreover, the $\mathrm{CNN}$ algorithm was initially designed to use the supervised method in the training procedures, and it is not expected a good performance if its structure is changed to an unsupervised fashion. Consequently, the room is still open for researchers to improve the performance of anomaly techniques to address Zero-day detection using supervised learning models.

\section{CONCLUSION AND FUTURE WORK}

In this paper, we proposed an anomaly detection-based technique using CNN and LSTM algorithms to extract the spatial and temporal features from the input data. The proposed model uses two regularization methods to improve the detection model capability in zero-day attacks. Moreover, we showed that our proposed CNN-LSTM approach outperformed either the single CNN or LSTM, which confirmed that the hybrid model has a strong potential to be used for real-time IDS. Additionally, using regularization techniques with the $\mathrm{CNN}$ algorithm enhanced the performance of the detection model compared to the standard CNN. For future work, we are planning to implement the proposed model in a real SDN system and test its performance in terms of throughput and latency. Further, we will test how the proposed model can work on different datasets.

\section{REFERENCES}

[1] Razan Abdulhammed, Hassan Musafer, Ali Alessa, Miad Faezipour, and Abdelshakour Abuzneid. 2019. Features dimensionality reduction approaches for machine learning based network intrusion detection. Electronics 8, 3 (2019), 322.

[2] Narmeen Zakaria Bawany, Jawwad A Shamsi, and Khaled Salah. 2017. DDoS attack detection and mitigation using SDN: methods, practices, and solutions Arabian fournal for Science and Engineering 42, 2 (2017), 425-441.

[3] Sarra BOUKRIA and Mohamed GUERROUMI. 2019. Intrusion detection system for SDN network using deep learning approach. In 2019 International Conference on Theoretical and Applicative Aspects of Computer Science (ICTAACS), Vol. 1 IEEE, 1-6.

[4] Ünal Çavuşoğlu. 2019. A new hybrid approach for intrusion detection using machine learning methods. Applied Intelligence 49, 7 (2019), 2735-2761.

[5] Mahmoud Said Elsayed, Nhien-An Le-Khac, Soumyabrata Dev, and Anca Delia Jurcut. [n. d.]. Ddosnet: A deep-learning model for detecting network attacks. In 21ST IEEE INTERNATIONAL SYMPOSIUM ON A WORLD OF WIRELESS, MOBILE AND MULTIMEDIA NETWORKS (IEEE WOWMOM 2020), Ireland. IEEE.

[6] Mahmoud Said Elsayed, Nhien-An Le-Khac, Soumyabrata Dev, and Anca Delia Jurcut. 2019. Machine-Learning Techniques for Detecting Attacks in SDN. In 2019 IEEE 7th International Conference on Computer Science and Network Technology (ICCSNT). IEEE, 277-281.

[7] Mahmoud Said Elsayed, Nhien-An Le-Khac, Soumyabrata Dev, and Anca Delia Jurcut. 2020. Detecting Abnormal Traffic in Large-Scale Networks. In 2020 IEEE International Symposium on Networks, Computers and Communications (ISNCC'20) IEEE.

[8] Mahmoud Said Elsayed, Nhien-An Le-Khac, and Anca D Jurcut. 2020. InSDN: A Novel SDN Intrusion Dataset. IEEE Access 8 (2020), 165263-165284.
[9] Mahmoud Said Elsayed, Nhien-An Le-Khac, and Anca Delia Jurcut. 2021. Dealing With COVID-19 Network Traffic Spikes [Cybercrime and Forensics]. IEEE Security \& Privacy 19, 1 (2021), 90-94.

[10] Ruben J Franklin, Vidyashree Dabbagol, et al. 2020. Anomaly Detection in Videos for Video Surveillance Applications Using Neural Networks. In 2020 Fourth International Conference on Inventive Systems and Control (ICISC). IEEE, 632-637.

[11] Anish Halimaa and K Sundarakantham. 2019. Machine learning based intrusion detection system. In 20193 rd International conference on trends in electronics and informatics (ICOEI). IEEE, 916-920.

[12] Hanan Hindy, Robert Atkinson, Christos Tachtatzis, Jean-Noël Colin, Ethan Bayne, and Xavier Bellekens. 2020. Utilising deep learning techniques for effective zero-day attack detection. Electronics 9, 10 (2020), 1684

[13] Sepp Hochreiter and Jürgen Schmidhuber. 1997. Long short-term memory. Neural computation 9, 8 (1997), 1735-1780.

[14] Dongxu Huang, Dejun Mu, Libin Yang, and Xiaoyan Cai. 2018. CoDetect: Financial fraud detection with anomaly feature detection. IEEE Access 6 (2018), 19161-19174.

[15] Ren-Hung Hwang, Min-Chun Peng, Chien-Wei Huang, Po-Ching Lin, and VanLinh Nguyen. 2020. An unsupervised deep learning model for early network traffic anomaly detection. IEEE Access 8 (2020), 30387-30399.

[16] Kubra Kalkan, Gurkan Gur, and Fatih Alagoz. 2017. Defense mechanisms against DDoS attacks in SDN environment. IEEE Communications Magazine 55, 9 (2017), 175-179.

[17] Murat Karakus and Arjan Durresi. 2017. Quality of service (QoS) in software defined networking (SDN): A survey. Fournal of Network and Computer Applications 80 (2017), 200-218.

[18] Zhengmin Kong, Yande Cui, Zhou Xia, and He Lv. 2019. Convolution and long short-term memory hybrid deep neural networks for remaining useful life prognostics. Applied Sciences 9, 19 (2019), 4156.

[19] Rocco Langone, Alfredo Cuzzocrea, and Nikolaos Skantzos. 2020. Interpretable Anomaly Prediction: Predicting anomalous behavior in industry 4.0 settings via regularized logistic regression tools. Data \& Knowledge Engineering (2020), 101850.

[20] Markus Ring, Sarah Wunderlich, Deniz Scheuring, Dieter Landes, and Andreas Hotho. 2019. A survey of network-based intrusion detection data sets. Computers \& Security (2019).

[21] Mahmoud Said Elsayed, Nhien-An Le-Khac, Soumyabrata Dev, and Anca Delia Jurcut. 2020. Network Anomaly Detection Using LSTM Based Autoencoder. In Proceedings of the 16th ACM Symposium on QoS and Security for Wireless and Mobile Networks. 37-45.

[22] Kazi Abu Taher, Billal Mohammed Yasin Jisan, and Md Mahbubur Rahman. 2019. Network intrusion detection using supervised machine learning technique with feature selection. In 2019 International Conference on Robotics, Electrical and Signal Processing Techniques (ICREST). IEEE, 643-646.

[23] Tuan A Tang, Lotfi Mhamdi, Des McLernon, Syed Ali Raza Zaidi, and Mounir Ghogho. 2016. Deep learning approach for network intrusion detection in software defined networking. In 2016 international conference on wireless networks and mobile communications (WINCOM). IEEE, 258-263.

[24] Tuan A Tang, Lotfi Mhamdi, Des McLernon, Syed Ali Raza Zaidi, and Mounir Ghogho. 2018. Deep recurrent neural network for intrusion detection in sdnbased networks. In 2018 4th IEEE Conference on Network Softwarization and Workshops (NetSoft). IEEE, 202-206.

[25] Arijit Ukil, Soma Bandyoapdhyay, Chetanya Puri, and Arpan Pal. 2016. IoT healthcare analytics: The importance of anomaly detection. In 2016 IEEE 30th international conference on advanced information networking and applications (AINA). IEEE, 994-997.

[26] Puming Wang, Laurence T Yang, Xin Nie, Zhian Ren, Jintao Li, and Liwei Kuang. 2020. Data-driven software defined network attack detection: State-of-the-art and perspectives. Information Sciences 513 (2020), 65-83.

[27] You-Chiun Wang and Siang-Yu You. 2018. An efficient route management framework for load balance and overhead reduction in SDN-based data center networks. IEEE Transactions on Network and Service Management 15, 4 (2018), 1422-1434.

[28] Yihan Xiao, Cheng Xing, Taining Zhang, and Zhongkai Zhao. 2019. An intrusion detection model based on feature reduction and convolutional neural networks. IEEE Access 7 (2019), 42210-42219. 